\title{
A Method for Nested PCR with Single Closed Reaction Tubes
}

\author{
Joseph Yourno
}

Wadsworth Center for Laboratories and Research, New York State Department of Health, Albany, New York 12201-0509

\begin{abstract}
Toward the goal of reducing diagnostic false positives while retaining high sensitivity, a closed-tube nested PCR procedure has been developed for detecting low-copy-number human immunodeficiency virus (HIV) gag target DNA sequences. Master mix for amplification 2, in a hanging gel matrix at the reaction tube top, remains sequestered from the reaction space of the tube during amplification 1. A severalfold excess of inner over outer primers is built into the procedure to assure the high sensitivity of nested PCR. The master mix for amplification 2 is then introduced into the reaction space by centrifugation, and the second amplification is performed as usual. The closed-tube nested procedure shows sensitivity approaching that of the open-tube control procedure, which detects a single copy of HIV gag target DNA at near-theoretical frequency, typically with microgram yields of specific amplification product.
\end{abstract}

$\mathbf{N}$ ested PCR greatly enhances sensitivity of detection of target nucleic acid sequences. ${ }^{(1)}$ The approach is based on, first, the amplification of an extended sequence and, second, the amplification of an internal sequence from the product of the first. The enhanced sensitivity of the method is achieved by controlling reaction conditions for each amplification to favor generation of the desired product. In addition to the usual considerations, most approaches require a severalfold excess of inner over outer primers in the second amplification for satisfactory results . Conventional methods accomplish this by amplifying only a small aliquot of the completed first amplification mixture after transfer to a new reaction tube for the second amplification. The increased sensitivity of nested PCR is bought at the price of potential false positives, because tubes containing high concentrations of the first amplification product must be opened and manipulated to set up the second amplification. A method for efficient nested PCR that eliminates the need for these manipulations would reduce the chance for false positives. The approach described herein is based on the use of a single reaction tube that remains closed for both amplifications after reagents are introduced for each. Reagents for the second amplification are sequestered and preserved in the reaction tube during the first amplification, and then introduced into the reaction mixture for the second amplification. A closed-tube nested procedure for detection of human immunodeficiency virus (HIV) gag sequences shows sensitivity approaching the range of the conventional procedure, which detects a single target DNA sequence with strong signal at near-theoretical frequency.

\section{MATERIALS AND METHODS}

Strategy

PCR amplification 1 with template DNA is set up in the usual fashion in reaction tubes and overlaid with mineral oil. Outer primers are used at reduced concentration. Master mix for amplification 2 is prepared in a melted, thin agarose gel matrix and introduced into the lidlock chamber of the reaction tubes. This mix contains inner primers at severalfold excess over outer primers for amplification 1 . The tube deadspace is filled with mineral oil to just below the lidlock line before tube closure. While the first amplification is run, the master mix for the second remains sequestered at the tube top, protected from extreme temperature changes to which the lower end of the tube is subjected. After the first amplification is completed, the reagents for the second amplification are introduced by brief centrifugation into the reaction space. The second amplification is then run as usual.

\section{Standard DNA Preparations}

Peripheral blood mononuclear cells (PBMC) from normal donors (American Red Cross) were isolated on Ficoll-Hypaque cushions, aliquoted, and stored in liquid nitrogen. DNA was prepared from thawed, washed PBMC in PCR buffer with nonionic detergents and protease $\mathrm{K}$ by the method of Higuchi. ${ }^{(2)}$ DNA from cells of the $8 \mathrm{E} 5$ cell line, which contain one integrated provirus per genome, ${ }^{(3)}$ was isolated similarly. White cell counts on PBMC and 8E5 cells were done by hemacytometer. Threefold dilutions of $8 \mathrm{E} 5$ DNA into normal PMBC DNA were made to establish standards containing from 33 copies of HIV provirus to 0 copies in 
$0.5 \mu \mathrm{g}$ total DNA per $40 \mu \mathrm{l}$ PCR buffer. DNA concentrations are based on $1 \mu \mathrm{g}$ DNA per 200,000 cells. Standard preparations in 1.5-ml gasketed screw cap tubes (Sarstedt) were boiled, snap-cooled in ice water, aliquoted into the same type of tube, and stored at $-20^{\circ} \mathrm{C}$.

\section{PCR Amplification}

A nested PCR procedure has been devised based on SK38 and SK39 ${ }^{(4)}$ as internal primers for HIV gag sequences. Reaction mix parameters are as follows. Amplification 1: Volume $50 \mu \mathrm{l}, \mathrm{MgCl}_{2}$ $2.5 \mathrm{~mm}$, outer primers (HPLC-purified, Operon) 12.5 pmoles each (SK380 5'-GAG AAC CAA GGG GAA GTG ACA TAG CAG G, 28-mer, HIV gag (HXB2) 687-712; SK390 5'-TAG AAC CGG TCT ACA TAG TCT CTA AAG GG, 29-mer, HIV gag (HXB2) 874-903), AmpliTaq 0.75 unit, deoxynucleoside triphosphates 200 $\mu \mathrm{M}$ each. Amplification 2: volume $75 \mu \mathrm{l}$, $\mathrm{MgCl}_{2} 2.5 \mathrm{mM}$, inner primers SK38 and SK39 (HPLC-purified, Operon) 75 pmoles each, AmpliTaq 1.03 unit (additional), deoxynucleoside triphosphates $200 \mu \mathrm{M}$ (additional).

Master mix for amplification 1 was prepared and aliquoted into $0.5-\mathrm{ml}$ Gene Amp reaction tubes (Perkin-Elmer Cetus) at $10.2 \mu l$ per tube. Four drops of mineral oil were introduced into each tube, and then $40 \mu \mathrm{l}$ of DNA preparation was added to the aqueous underphase. Master mix for reaction 2 was prepared and experiments from this point followed one of two variations, each based on use of $25 \mu \mathrm{l}$ of master mix 2 for amplification 2 .

\section{Control Open-tube Procedure}

After PCR amplification 1, tubes were opened with flamed forceps, aqueous master mix 2 (stored at $4^{\circ} \mathrm{C}$ ) was introduced into the aqueous layer, and the tubes were resealed for amplification 2 .

\section{Closed-tube Procedure with Master Mix 2 Sequestered in Agarose Gel Matrix}

Before sealing the reaction tubes for amplification 1, master mix 2 in melted $0.25 \%$ agarose (Seakem) at $70-80^{\circ} \mathrm{C}$ was aliquoted with a positive-displacement pipette into the lid-lock chamber of the reaction tube. This master mix was prepared from stock aliquots of $0.375 \%$ aga- rose in $1.5 \times$ PCR buffer, and stored at $-20^{\circ} \mathrm{C}$ in $1.5-\mathrm{ml}$ screw-cap tubes. For each experiment, an aliquot of buffer/gel in the storage tube was melted by boiling 3-5 min. When the tube reached $70-80^{\circ} \mathrm{C}$, it was removed from the water bath and the other components of master mix 2 were added at room temperature directly to the melted gel/buffer. The contents were mixed, reincubated $2-4 \mathrm{~min}$ at $70-80^{\circ} \mathrm{C}$ in the resealed screw-cap tubes, and then aliquoted into reaction-tube lids at room temperature. A small drop of mineral oil (about $15 \mu \mathrm{l}$ ) was layered over the solidified gel. Mineral oil was added to each tube to just under the lid-lock line and the tube was sealed, leaving a small airspace in the lidlock chamber below the adherent gel.

The effect of cooling on preserving master mix 2 in the gel matrix during amplification 1 was examined by placing a flat-bottomed aluminum block filled with ice flush over the reaction tubes in the cycler, which was covered with a replacement lid. Ice was replaced every 30 min, during the $55^{\circ} \mathrm{C}$ plateau of the cycle. Since the actual extent of cooling achieved was indeterminate, this parameter was examined more exactly by mock closed-tube nested PCR. Similarly prepared reaction tubes, containing master mix 2 gel in the lid-lock chamber and filled as described above with mineral oil, were stored at $4^{\circ} \mathrm{C}$. Then the lids were exchanged with those of experimental reaction tubes after amplification 1 .

In all cases the gel containing master mix 2 was introduced into the reaction mixture by brief centrifugation at 12,500 $\times g(10-60 \mathrm{sec})$. Tubes were then inverted to mix the contents, recentrifuged as above, and subjected to the second amplification.

For each amplification, tubes were placed in the block of a Perkin-Elmer Cetus thermal cycler set of $94^{\circ} \mathrm{C}$, incubated at $95^{\circ} \mathrm{C}$ for $60 \mathrm{sec}$, and then subjected to 35 thermal cycles $\left(95^{\circ} \mathrm{C} \times 60 \mathrm{sec}\right.$, $\left.55^{\circ} \mathrm{C} \times 60 \mathrm{sec}, 60^{\circ} \mathrm{C} \times 60 \mathrm{sec}\right)$, followed by an extension step $\left(60^{\circ} \mathrm{C} \times 10 \mathrm{~min}\right)$.

Unnested control amplifications were also performed with SK380 and SK390 or SK38 and SK39 as primers. Reaction mix parameters were as given above for amplification 1, except that primer content was 50 pmoles each per $50 \mu$ l of final reaction volume. Provirus input ranged from 0 to 100 copies per tube in PBMC DNA and 1000 copies in PCR buffer. Am- plifications followed the protocol given above for 35 cycles and/or 70 cycles.

\section{Analysis of PCR Product}

PCR amplification products were analyzed by liquid hybridization with ${ }^{32} \mathrm{P}$ end-labeled SK19 probe. ${ }^{(4)}$ An aliquot of $5 \mu$ l of each specimen was hybridized with $100,000-115,000 \mathrm{cpm}$ of SK19 probe in a final volume of $9-10 \mu l$ and at $150 \mathrm{~mm} \mathrm{NaCl}$. The hybridization mixture was diluted with an equal volume of $2 \times$ stop buffer and the entire contents were subjected to PAGE in $8 \%$ gels. X-ray film (Kodak XAR-2) was exposed to completed gels in cassettes equipped with intensifying screens for 4-16 hr at room temperature, or at $-70^{\circ} \mathrm{C}$ for maximum sensitivity.

To estimate SK38/39 amplification product yield in weak to moderate positives, signal on autoradiographs was compared to that from serial dilutions of a nested strong positive (1-2 $\mu \mathrm{g}$ product) on autoradiographs given equal exposure. The strong positive was diluted in PBMC DNA $(0.5 \mu \mathrm{g}$ per $50 \mu \mathrm{l}$ of PCR buffer) $3.7 \times, 11 \times, 33 \times, 100 \times$, and then 10 -fold serially up to $1 \times 10^{9} \times$. Undiluted specimen and dilutions thereof were hybridized with the standard amount of SK19 radiolabel for autoradiography. It was found that: (1) there was a $3.7 \times$ to $11 \times$ excess of product for complete uptake of probe and (2) product was detectable down to $10^{-5} \times$ concentration (i.e., at $1-2 \times 10^{-5} \mu \mathrm{g}$ ).

The SK38/39 gag amplification product of $115 \mathrm{bp}$ was also detected directly by agarose gel electrophoresis and ethidium bromide fluorescence. An aliquot of $18 \mu$ l of specimen in $1 \times$ stop buffer was run in a $1 \%$ Seakem, $2 \%$ Nusieve gel $(1 \times$ Tris-borate-EDTA, $\mathrm{pH} \mathrm{8.3)} \mathrm{at} 5 \mathrm{~V} / \mathrm{cm}$ for about 2 hr. A $\phi X 174$ HaeIII DNA digest was used for molecular weight markers. For a semiquantitative estimate of amplification product yield, $10 \mu$ l of a $5 \times$ dilution of each positive specimen in $1 \times$ stop buffer was run similarly against serial dilutions of the $\phi X 174$ HaeIII DNA digest at known concentrations. Band strength of diluted amplification product was compared to this standard and bracketed between the nearest greater and lesser dilutions. This method proved capable of detecting $\geqslant 1 / 20 \mu \mathrm{g}$ of SK38/39 amplification product.

Modified Southern blots were prepared from amplified material run as 
above at $10 \mu \mathrm{l}$ per specimen and transferred by capillary action with $0.4 \mathrm{~N}$ $\mathrm{NaOH}$ from the agarose gel onto a nylon membrane $^{(6)}$ (Zeta-Probe). The membrane was rinsed with $6 \times$ SSC and irradiated while moist with 0.36 Joules $/ \mathrm{cm}^{2}$ of UV light (Stratalinker). All steps of the procedure described below up to the last wash were performed at room temperature with the hybridization mixture under constant agitation on a platform shaker set at $80 \mathrm{cycles} / \mathrm{min}$. The membrane was prehybridized in $75 \mathrm{ml}$ of $6 \times$ SSC $+1 \%$ SDS for $1 \mathrm{hr}$, then hybridized in $10 \mathrm{ml}$ of the same medium containing about $1 \times 10^{6} \mathrm{cpm}$ of ${ }^{32} \mathrm{P}$ end-labeled SK19 probe $^{(4)}$ for $1 \mathrm{hr}$. The membrane was washed twice with $75 \mathrm{ml}$ of $6 \times$ SSC for $15 \mathrm{~min}$. The washing was continued as above with $2 \times$ SSC, $0.67 \times$ SSC, and $2 \times$ SSC $+1 \%$ SDS. A final wash step was done with $2 \times$ SSC $+1 \%$ SSC at $37^{\circ} \mathrm{C}$. XAR-2 film was exposed to the membrane in a film cassette for $2 \mathrm{hr}$ at room temperature.

\section{RESULTS}

DNA standards were first checked by the open-tube nested PCR control procedure. Liquid hybridization with radiolabeled SK19 probe and autoradiography detected 32 strong positives (Fig. 1) and 3 weak positives (Fig. 2). The weak positives were all from single-copy provirus specimens. All expected negatives showed no detectable signal after film exposure up to $16 \mathrm{hr}$ at $-70^{\circ} \mathrm{C}$. These results are within the expected values at each provirus copy number tested, from 33 to zero per tube (Table 1, Series 1). In particular, with lowest-copy-number positives, the fraction of positives/total tested decreased in a manner consistent with the cumulative Poisson distribution for provirus per specimen. At 1.2 copies per tube, 13 positives were observed per 16 specimens (95\% confidence interval, 5.8-14.1 positives/16 total); at 0.4 copies per tube, 3 positives were observed per 12 specimens (95\% confidence interval, $0.5-7.5$ positives $/ 12$ total). These results suggest that the nested PCR procedure picks up a single copy of target DNA at near-theoretical frequency. The yield of SK38/39 amplification product in strong positives was sufficient to take up all probe on liquid hybridization and to allow its direct detection by agarose gel electrophoresis and ethidium bromide fluorescence (Fig. 3). A yield of 1-2 $\mu$ g

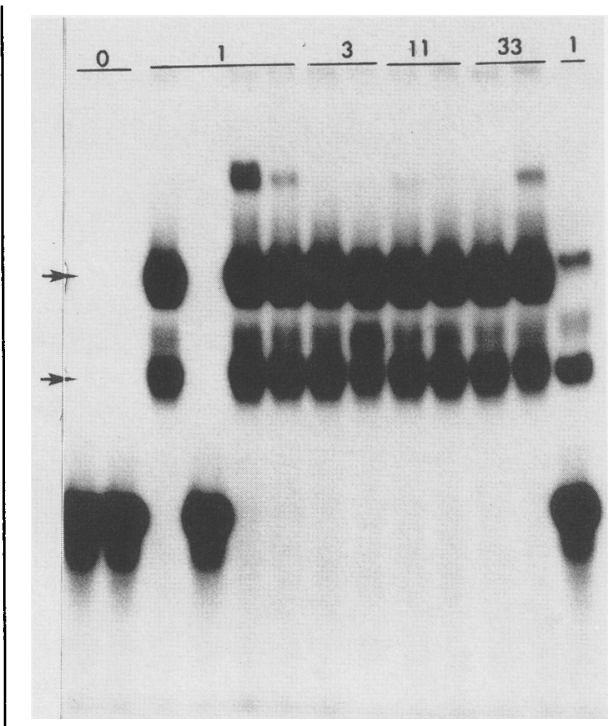

FIGURE 1 Autoradiographic detection of SK38/39 amplification product after hybridization to radiolabeled SK19 probe and PAGE using closed-tube nested procedure and ambient cycler temperature. Film exposure was for $4 \mathrm{hr}$ at room temperature. Provirus copy number of duplicate specimens (underlined) is indicated above the lanes. Fast-migrating probe is not taken up by negative specimens (lanes 1,2, and 4). Strong positives from 1.0 to 33 copies of provirus take up probe completely, forming two major hybrid bands and one or more weaker bands (lanes 3-12, arrows). These represent heteroduplexes, triplexes, quadriplexes, etc. A moderate positive from a single-copy specimen takes up probe partially (lane 13). Titration experiments bracket the amplification product concentration in this and another nested single-copy moderate positive between 1 and $3 \%$ that of strong positives.

product per specimen was estimated by this procedure, representing an amplification efficiency of 5-10\% based on 16.7 $\mu \mathrm{g}$ equivalent of DNA per $200 \mu \mathrm{M}$ deoxynucleoside triphosphates in $75 \mu$ l of reaction mixture. There was no obvious gradient of product yield from singlecopy to multiple-copy strong positives. By autoradiographic titration experiments, the yield of SK38/39 amplification product in all three weak positives was bracketed between $10^{-4} \times$ and $10^{-3} \times$ that of strong positives. The cause of these nesting failures is not clear, but signal strength on autoradiography was in the range of single-copy positives detected by unnested amplification with SK38 and 39 primers (see below). By gel/fluorescence, virtually all specimens, including these, showed the usual pattern of nonspecific bands, indicating that amplification 1 proceeded normally (the one exception is discussed below).

The effect of indeterminate amounts of residual enzyme and deoxynucleoside triphosphates from amplification 1 on amplification 2 as set up here was checked by running a conventional procedure with the same series of duplicate DNA standards. Here a $12.5-\mu 1$ aliquot of completed amplification 1 reaction mixture (SK380 and 390 primers at 50 pmoles each in $50 \mu \mathrm{l}$ ) was transferred to a new reaction tube for amplification 2 (SK38 and 39 primers at 75 pmoles each in $75 \mu \mathrm{l})$. In this manner, enzyme (1.13 units per tube) and deoxynucleoside triphosphates ( $200 \mu \mathrm{m}$ each) were closely controlled. Similar yields of SK38/39 amplification product were found from this modification, estimated at 1-2 $\mu \mathrm{g}$ per positive in the entire range tested, from 1 to 1000 provirus copies (data not shown).

The identity of the 115-bp SK38/39 amplification product in selected specimens from the nested procedure was confirmed on Southern blots, where it was specifically and strongly visualized with radiolabeled SK19 probe (Figs. 4C and $5 \mathrm{C}$ ) and was indistinguishable from product of the unnested amplification 2 with SK38 and 39 primers (Figs. 4B and 5B).

The closed-tube nested procedure showed sensitivity approaching that of the open-tube control (Table 1, Series 2; Figs 1, 2, and 3). With one exception, observed positives were within the expected range for the cumulative Poisson distribution at the calculated copy number of provirus (13 positives/14 singlecopy specimens run at ambient cycler temperature; $95 \%$ confidence interval, 4.8-12.7 positives/14 total). Autoradiography showed 68 strong positives and 3 moderate positives. The strong positives were readily detectable by gel/fluorescence but the moderate positives were missed by this technique. Two of these stemmed from single-copy specimens. Product yield of each was bracketed between 1 and $3 \%$ that of strong positives by autoradiographic titration. The third moderate positive was from an 11-copy specimen, product yield estimated at 3-9\%, which showed an anomalous pattern of bands by gel/fluorescence (data not shown). Among strong positives, SK38/39 product yield was typically in 


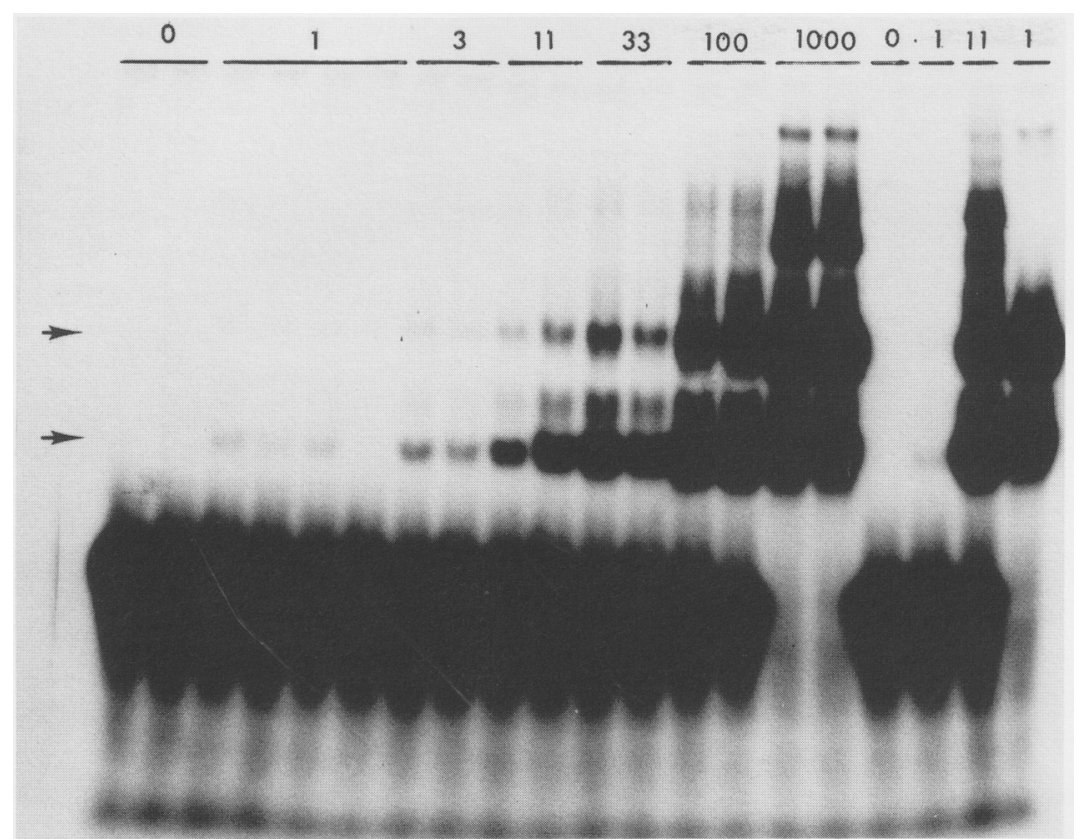

FIGURE 2 Autoradiographic detection of SK38/39 amplification product hybridized to SK19 probe. Unnested amplification for 70 cycles (from left, lanes 1-16) and representative nested amplifications (lanes 17-20). Film exposure was for $16 \mathrm{hr}$ at room temperature. Provirus copy number of duplicate specimens is given above lanes. See Fig. 1 legend for further details. In the unnested amplification, SK38/39 product is weakly detectable down to the single-copy level (arrows). The signal from lane 6 was judged indeterminate and was not improved above background after $16 \mathrm{hr}$ of film exposure at $-70^{\circ} \mathrm{C}$ (data not shown). A weak single-copy positive from the open-tube nested procedure is shown in lane 18 . The SK38/39 product concentration in this and all other weak single-copy positives from the nested and unnested procedures was estimated at $10^{-4} \times$ to $10^{-3} \times$ that of strong positives. A moderate positive from the closed-tube nested procedure, from a specimen with 11 copies of provirus which gave anomalous bands on agarose gel electrophoresis, is shown in lane 19. Estimated product is between 3 and $9 \%$ that of strong positives. Lane 20 shows a strong single-copy positive from the nested procedure for comparison.

the range achieved by the open-tube control method, i.e., $1-2 \mu \mathrm{g}$ per tube. The open-tube procedure, however, invariably gave equal or somewhat greater

yields within the bracketed values as judged by band strength.

There was a tendency toward formation of apparent primer dimers in ampli-

TABLE 1 Closed-tube Nested PCR-Summary of Results

\begin{tabular}{|c|c|c|c|c|c|}
\hline \multirow{3}{*}{$\begin{array}{l}\text { Provirus }^{a} \\
\text { (copies per } \\
\text { reaction) }\end{array}$} & \multirow{3}{*}{$\begin{array}{l}\frac{\text { Series } 1^{\mathrm{b}}}{\text { Open-tube }} \\
\text { control }\end{array}$} & \multicolumn{4}{|c|}{ Series $2^{b}$} \\
\hline & & \multirow{2}{*}{$\begin{array}{l}\text { open-tube } \\
\text { control }\end{array}$} & \multicolumn{3}{|c|}{ Agarose gel matrix at } \\
\hline & & & $4^{\circ} \mathrm{C}$ storage & cool & ambient temperature \\
\hline 0 & $0 / 10$ & $0 / 6$ & $0 / 6$ & $0 / 6$ & $0 / 6$ \\
\hline 0.4 & $3 / 12$ & & & & \\
\hline 1.0 & & $11 / 13$ & $8 / 11(1++)^{d}$ & $6 / 12$ & $13 / 14^{\mathrm{c}}(1++)$ \\
\hline 1.2 & $13 / 16(3+)^{d}$ & & & & \\
\hline 3.7 & $10 / 10$ & $6 / 6$ & $6 / 6$ & $4 / 4$ & $6 / 6$ \\
\hline 11 & $5 / 5$ & $5 / 5$ & $5 / 5$ & $4 / 4(1++)$ & $6 / 6$ \\
\hline 33 & $4 / 4$ & $5 / 5$ & $4 / 4$ & $4 / 4$ & $5 / 5$ \\
\hline
\end{tabular}

${ }^{\text {a }}$ The standard deviation for provirus copy number (based on 8E5 cell count) is $\pm 13 \%$.

b,c Positives/total. In each case except c, the fraction of observed positives falls within the $95 \%$ confidence interval for expected positives, based on the cumulative Poisson distribution of provirus per reaction volume at the calculated mean copy number.

${ }^{\mathrm{d}}$ Signal strength of positives detectable only by autoradiography is given as + (weak) and ++ (moderate). All other positives were strong $(++++$ ), i.e., detectable by gel electrophoresis and ethidium bromide fluorescence as well as by autoradiography.

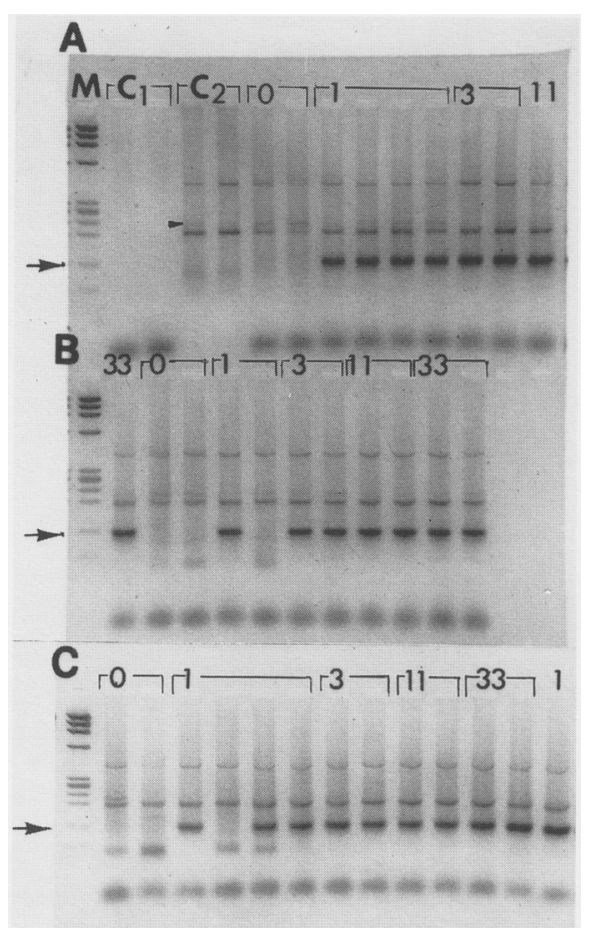

FIGURE 3 Agarose gel electrophoresis of SK38/39 amplification product from nested PCR, direct visualization by ethidium bromide fluorescence, negative image. $(A)$ Opentube control procedure: (M) $\phi X 174$ HaeIII molecular-weight markers; $\left(\mathrm{C}_{1}\right)$ unamplified genome DNA control, containing reagents for amplification 1 and $2 ;\left(\mathrm{C}_{2}\right)$ amplification 1 control; (0) zero copies provirus; (1) 1 copy; (3) 3.7 copies; (11) 11 copies. Duplicate specimens are bracketed. $(B)$ Open-tube control procedure continued: (lane 1) molecularweight markers as A; (33) 33 copies. Mock closed-tube procedure, master mix 2 stored at $4^{\circ} \mathrm{C}$, provirus copy number as above. (C) Closed-tube procedure, master mix 2 at cycler ambient temperature during amplification 1, provirus copy number and molecular-weight markers as $A$. The arrow at the left of each gel indicates the prominent SK38/39 amplification product of $115 \mathrm{bp}$, running slightly faster than the 118-bp marker band in the $\phi X 174$ HaeIII digest. A moderate single-copy positive not detected by this method is shown in $B$, lane 6 from left. The arrowhead by lane 3 from left $(A)$ indicates the position of the SK380/390 amplification product of $216 \mathrm{bp}$, which runs with the weak nonspecific band visible in most lanes but which itself is not visible at these low-copy levels.

fication 2 of the closed-tube procedure with negative or low-copy-number specimens (Fig. 3). Occasional weak bands of this size were also seen with highercopy-number specimens, in the presence of a prominent SK38/39 amplification product (data not shown). These results 


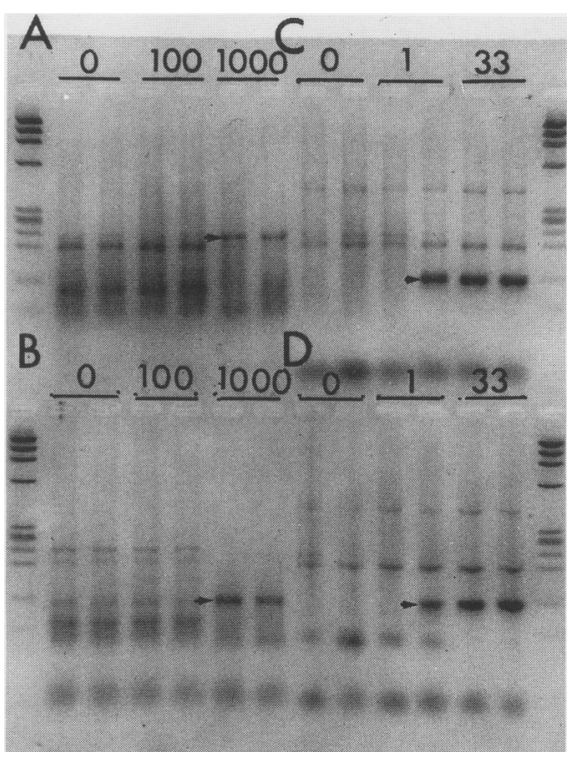

FIGURE 4 Agarose gel electrophoresis of amplification product from nested procedure and unnested amplifications 1 and 2, direct visualization by ethidium bromide fluorescence, negative image. Molecular-weight markers in each outer lane are as described in Fig. 3 legend. $(A)$ Amplification 1, 70 cycles. Duplicate specimens at 0,100 , and 1000 copies of provirus were run as indicated by underlined numbers above gel lanes. The 216-bp SK380/390 amplification product (arrowhead) is clearly detectable only in the 1000-copy specimens, amplified without normal genomic DNA background. This overlaps a nonspecific band, which is visible in lower-copynumber specimens. (B) Amplification 2, 70 cycles. Duplicate specimens at 0,100 , and 1000 copies of provirus. The 115-bp SK38/39 amplification product (arrowhead), yield 1/3$2 / 3 \mu \mathrm{g}$ per specimen, is clearly detectable only in the 1000-copy specimens, amplified without normal genomic DNA background. This runs slightly behind the nonspecific band seen in lower-copy-number specimens. (C) Open-tube nested PCR control. Duplicate specimens at $0,1.0$ (1 positive, 1 negative), and 33 copies of provirus. Arrowhead indicates SK38/39 product band in positive specimens, yield 1-2 $\mu \mathrm{g}$ each. (D) Closed-tube nested PCR, ambient cycler temperature. Duplicate specimens at $0,1.0$ ( 1 positive and 1 negative), and 33 copies of provirus. Arrowhead indicates $S K 38 / 39$ product band in positive specimens, yield $1 / 2-1 \mu \mathrm{g}$ in single-copy positive, 1-2 $\mu \mathrm{g}$ in 33 copy specimens.

suggest some competitive primer dimer formation in the method as performed. Master mix 2 subjected to ambient cycler temperature in general yielded as much SK38/39 amplification product as mix cooled or refrigerated during the first

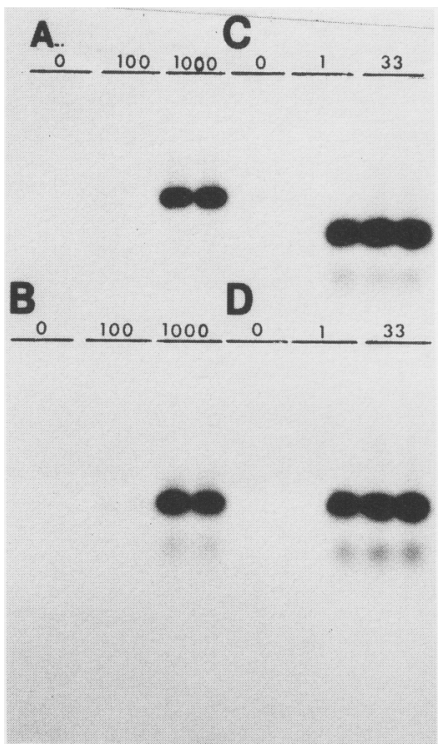

FIGURE 5 Modified Southern blot from agarose gel run shown in Fig. 4. Autoradiography for $2 \mathrm{hr}$ at room temperature (see Fig. 4 legend for further details). Radiolabeled SK19 probe confirms identity of 216-bp SK380/390 and 115-bp SK38/39 amplification products. Some signal is also seen in the SK38/39 positives at the approximate position of single-stranded product chains. Weak signal is detectable in 100-copy specimens amplified by the unnested procedure with SK38 and 39 primers. The several distinct nonspecific products produced by each amplification and visualized on gel/fluorescence in Figs. 3 and 4 are not detected by SK19 probe.

amplification of the closed-tube procedure, but the yield of occasional specimens was somewhat lower (0.5-1 $\mu \mathrm{g}$ per strong positive; see Fig. 4D). All strong positives from the closed-tube procedure using cooling of master mix 2 or refrigeration gave yields in the 1 - to $2-\mu \mathrm{g}$ range. These results suggest that sensitivity of the closed-tube nested procedure may be enhanced somewhat by cooling of master mix 2 during amplification 1 . When examined on Southern blots, the 115-bp SK38/39 amplification product from the closed-tube nested procedure showed the expected characteristics (Fig. 5D).

The ability of the unnested component amplifications 1 and 2 to detect target proviral DNA was compared with that of the nested amplification procedure. Unnested amplification 1 with SK380 and 390 primers for 70 cycles detected down to 11 to 3.7 copies of provirus with weak signal on autoradiography (data now shown). The predicted 216-bp SK380/390 amplification product was visualized only from 1000-provirus-copy specimens on gel electrophoresis and Southern blots (Figs. 4A and 5A). Unnested amplification 2 with SK38 and 39 primers, whether run for 35 cycles ( 3 positives $/ 4$ total) or for 70 cycles ( 3 positives, 1 indeterminate $/ 4$ total), detected down to a single copy of provirus with weak to indeterminate signal on autoradiography (Fig. 2). There was no significant improvement in signal strength gained by the additional cycling, except for 1000-copy specimens, where a stronger 115 -bp SK38/39 product band was visualized by gel/fluorescence and Southern blotting (Figs. 4B and 5B).

Comparative efficiencies of amplification of the nested and unnested procedures were estimated by three approaches. First, band strengths of amplification products from known copynumber specimens were compared on ethidium bromide-stained agarose gels and on derivative Southern blots. SK38/ 39 amplification product was strongly visible in the nested single-copy positives ( $1 / 7.5$ of specimen run), but this product and the SK380/390 product was found at equivalent to somewhat lesser concentrations only in the unnested 1000-copy specimens ( $1 / 5$ of specimen run) (Figs. 4 and 5). The 1000-copy specimens were run without normal PBMC DNA as diluent to visualize specific amplification product better and to obviate potential inhibition of amplification of the target proviral DNA sequences. These results suggest the amplifying power of the nested procedures is $\$ 1500 \times$ that of the unnested component amplifications 1 and 2. Second, the amounts of SK38/39 amplification product from nested and unnested procedures necessary to take up SK19 probe completely on liquid hybridization and autoradiography were compared in titration experiments. SK38/39 product from 70 cycles of unnested amplification took up probe completely between 100 and 1000 provirus copies per specimen (1/10 of specimen run, Fig. 2), whereas the nested product was $3.7 \times$ to $11 \times$ in excess of the amount necessary to accomplish the same (1/15 of specimen run, data not shown). These results suggest $600-16,000 \times$ greater amplifying power of the nested procedures over the unnested. Visual estimation of the data is most consistent with a several thousandfold increase. Third, as dis- 
cussed above, a nested strong positive required 1000-10,000 $\times$ dilution to match the attenuated signal of single-copy positives from the unnested amplification. As for moderate positives from the closed-tube nested procedure, titration experiments showed that these were at least $10-100 \times$ stronger than those of the unnested procedure at equivalent provirus copy number.

\section{Discussion}

A closed-tube nested PCR procedure has been developed that shows high sensitivity approaching that of the open-tube procedure. A single copy of HIV gag target DNA is detected at near-theoretical frequency, typically with microgram yields of SK38/39 amplification product. Although it has been developed to detect HIV gag sequences, this procedure could be adapted for amplification of other target DNA sequences as well. As designed here for detection purposes, the singletube protocol incorporates some indeterminate excess of reagents in amplification 2. This results in no loss of amplifying power. For amplification and more exact analysis of target sequences, e.g., by DNA sequencing, it may be advisable to drop deoxynucleoside triphosphate input for amplification 1 so that these do not exceed $200 \mu \mathrm{M}$ for amplification 2 , or otherwise modify reaction parameters, to minimize potential incorporation errors by Taq polymerase. ${ }^{(7)}$

The unnested amplification with SK38 and 39 primers also showed the ability to detect a single copy of provirus, but with marginal signal. While its reproducibility has not been studied systematically in this laboratory, unnested amplification using a somewhat different cycler program ${ }^{(5)}$ picked up 3.7-1.2 copies of provirus. The closed-tube nested procedure typically amplifies the target DNA sequence several hundredfold to a thousandfold more than the unnested procedure without significantly increasing the chance for false positives. Hence, it confers particular advantages in detection and analysis of rare or uncommon target sequences, where the ability of even a highly efficient unnested procedure, ${ }^{(4,5)}$ adapted for use here, is taxed.

This study is based on a prototype design for closed-tube nested PCR where liquid master mix 2 is retained in the lidlock chamber of the reaction tube with an inert membrane (parafilm, Teflon tape) and cooled during amplification 1, then discharged as above for amplification 2. The initial design verified the principle of the approach but presented significant leakage problems. Consequently, the present method was developed for better retention and sequestering of master mix 2 during amplification 1. Most of the deadspace of the reaction tube is filled with mineral oil, leaving a small insulating air lock in the lid-lock chamber under the master mix 2 gel. With this modification, sensitivity approaching that of the open-tube procedure has been attained, even when master mix 2 is subjected to the ambient temperature of the cycler during amplification 1. Occasional partial or complete nesting failures were observed both with the open-tube control and the closed-tube method. The cause of these failures remains to be determined. One may speculate that the weak positives from single-copy specimens reflect failure of amplification 1 due to episodic, inadequate unmasking of longer proviral DNA sequences after denaturation of genomic DNA. The moderate positives generated by the closed-tube method speak for inherent technical failures and are being dealt with by ongoing refinements. Although the number of experiments is limited, the more consistently high yields of SK38/39 amplification product with refrigerated or cooled master mix 2 suggest that cooling does improve sensitivity toward that of the control procedure. A cycler incorporating an efficient cooling plate flush across the reaction tube tops during amplification 1 would optimize results. The experimental design that has evolved here is based more on materials than conceptual constraints. For instance, any number of approaches based on the same principle may be visualized that trap master mix 2 in an appropriately engineered reaction tube chamber or suitable inert barrier.

\section{ACKNOWLEDGMENTS}

Evelyn Keller of the Virology Laboratory kindly supplied 8E5 cells used here; Leo Grady of the same laboratory furnished space for preparation and storage of PCR reagents. The dedicated typing assistance of Ms. Margaret Prescott is also acknowledged.

\section{REFERENCES}

1. Mullis, K.B. and F.A. Faloona. 1987. Specific synthesis of DNA in vitro via a polymerase catalyzed chain reaction. Methods Enzymol. 155: 335-350.

2. Higuchi, R. 1989. Rapid, efficient DNA extraction for PCR from cells or blood. Amplifications 2: 1-3.

3. Gendelman, H.E., T.S. Theodore, R. Willey et al. 1987. Molecular characterization of a polymerase mutant human immunodeficiency virus. Virology 160: 232-239.

4. Ou, C.Y., S. Kwok, S.W. Mitchell et al. 1988. DNA amplification for direct detection of HIV-1 in DNA of peripheral blood mononuclear cells. Science 239: 295-297.

5. Rogers, M.F., C.Y. Ou, M. Rayfield et al. 1989. Use of the polymerase chain reaction for the detection of the proviral sequences of the human immunodeficiency virus in infants born to serpositive mothers. N. Engl. J. Med. 320: 1649-1654.

6. Reed, K.C. and D.A. Mann. 1985. Rapid transfer of DNA from agarose gels to nylon membranes. Nucleic Acids Res. 13: 7207-7221.

7. Saiki, R.K. 1989. The design and optimization of the PCR. In PCR technology: Principles and applications for DNA amplification (ed. H.A. Erlich), pp. 7-16. Stockton Press, New York.

Received January 30, 1992; accepted in revised form May 11, 1992. 


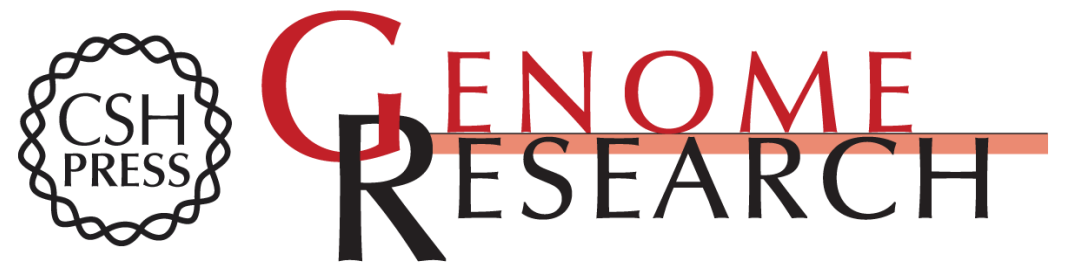

\section{A method for nested PCR with single closed reaction tubes.}

J Yourno

Genome Res. 1992 2: 60-65

Access the most recent version at doi:10.1101/gr.2.1.60

$\begin{array}{ll}\text { References } & \text { This article cites } 6 \text { articles, } 1 \text { of which can be accessed free at: } \\ \text { http://genome.cshlp.org/content/2/1/60.full.html\#ref-list-1 }\end{array}$

\section{License}

Email Alerting Receive free email alerts when new articles cite this article - sign up in the box at the Service top right corner of the article or click here.

\section{Affordable, Accurate Sequencing.}

To subscribe to Genome Research go to: https://genome.cshlp.org/subscriptions 